\title{
Quantum droplets of bosonic mixtures in a one-dimensional optical lattice
}

\author{
Ivan Morera $\odot,{ }^{1,2}$ Grigori E. Astrakharchik, ${ }^{3}$ Artur Polls, ${ }^{1,2}$ and Bruno Juliá-Díaz $\circledast^{1,2,4}$ \\ ${ }^{1}$ Departament de Física Quàntica i Astrofísica, Facultat de Física, Universitat de Barcelona, E-08028 Barcelona, Spain \\ ${ }^{2}$ Institut de Ciències del Cosmos, Universitat de Barcelona, ICCUB, Martí i Franquès 1, E-08028 Barcelona, Spain \\ ${ }^{3}$ Departament de Física, Universitat Politècnica de Catalunya, Campus Nord B4-B5, E-08034 Barcelona, Spain \\ ${ }^{4}$ ICFO-Institut de Ciencies Fotoniques, The Barcelona Institute of Science and Technology, E-08860 Castelldefels (Barcelona), Spain
}

(Received 14 January 2020; accepted 18 March 2020; published 9 April 2020)

\begin{abstract}
We demonstrate the existence of quantum droplets in two-component one-dimensional Bose-Hubbard chains. The droplets exist for any strength of repulsive intraspecies interactions provided they are balanced by comparable attractive interspecies interactions. The ground-state phase diagram is presented and the different phases are characterized by examining the density profile and off-diagonal one- and two-body correlation functions. A rich variety of phases is found, including atomic superfluid gases, atomic superfluid droplets, pair superfluid droplets, pair superfluid gases, and a Mott-insulator phase. A parameter region prone to be experimentally explored is identified, where the average population per site is lower than three atoms, thus avoiding three-body losses. Finally, the bipartite entanglement of the droplets is found to have a nontrivial dependence on the number of particles.
\end{abstract}

DOI: 10.1103/PhysRevResearch.2.022008

Ultracold gases trapped in optical lattices provide highly controllable setups which nowadays implement versatile quantum simulators for quantum many-body problems [1,2]. A prominent example is the experimental observations of a quantum zero-temperature phase transition (QPT) [3] between a weakly interacting superfluid Bose gas and a strongly interacting Mott insulator in three [4,5] and one dimensions [6].

Recently, a whole new class of ultradilute dropletlike quantum liquids has been studied both theoretically [7-10] and experimentally [11-17]. Such quantum droplets are self-bound objects and are capable of existing with no external trapping, similarly to the case of helium droplets [18]. The crucial difference is that the ultracold atoms provide an unprecedented control over the tunability of interactions and geometry of the system. The droplets were first produced in Bose gases with dipolar interactions $[11-13,19]$ and afterwards in binary bosonic mixtures with contactlike interactions [14-17]. The observed equilibrium density can be eight orders of magnitude smaller than in liquid helium, due to an almost exact compensation between mean-field repulsion and attraction. Moreover, it was evidenced [7] that the existence of the quantum droplets itself is a beyond mean-field effect as on the mean-field level the system collapses.

Arguably, the one-dimensional (1D) case is very promising. On one hand, in 1D quantum effects are enhanced, and, on the other hand, stability is increased due to the suppression of three-body losses [19-22]. Contrary to the 3D case, the

Published by the American Physical Society under the terms of the Creative Commons Attribution 4.0 International license. Further distribution of this work must maintain attribution to the author(s) and the published article's title, journal citation, and DOI. quantum droplets appear in the regime where at the meanfield level the system is on average repulsive and predicts a stable gas [23]. In this case, quantum fluctuations result in an effective attraction which is able to liquefy the system for an arbitrary number of particles [24].

In this Rapid Communication we go one step further and demonstrate the existence of quantum droplets in 1D Bose-Hubbard mixtures for both small and large interaction strengths. Computing the ground state with density-matrix renormalization group (DMRG) methods using TENPY [25], we obtain the phase diagram of the system in the relevant parameter region where droplets can be produced. A very rich phase diagram is obtained, with atomic and pair superfluids appearing in both droplet and gas phases. This exciting scenario is found to be within imminent reach with current experimental setups.

Two-component Bose-Hubbard model. We study a bosonic mixture with short-range interactions loaded into a high 1D optical lattice at zero temperature described by the BoseHubbard Hamiltonian [2]

$$
\begin{aligned}
H= & -t \sum_{i} \sum_{\alpha=A, B}\left(\hat{b}_{i, \alpha}^{\dagger} \hat{b}_{i+1, \alpha}+\text { H.c. }\right) \\
& +\frac{U}{2} \sum_{i} \sum_{\alpha=A, B}\left[\hat{n}_{i, \alpha}\left(\hat{n}_{i, \alpha}-1\right)\right]+U_{A B} \sum_{i} \hat{n}_{i A} \hat{n}_{i B},
\end{aligned}
$$

where $\hat{b}_{i \alpha}\left(\hat{b}_{i \alpha}^{\dagger}\right)$ are the annihilation (creation) bosonic operators at site $i=1, \ldots, L$ for species $\alpha=A, B$, respectively, and $\hat{n}_{i \alpha}$ are their corresponding number operators. We assume an equal tunneling strength, $t>0$, and repulsive intraspecies interaction strength, $U>0$, for both components. For the rest of the work we set the energy scale to the tunneling strength $t$, which is kept fixed, and work with an equal number of bosons of both species, $N_{A}=N_{B} \equiv N / 2$. For convenience, 

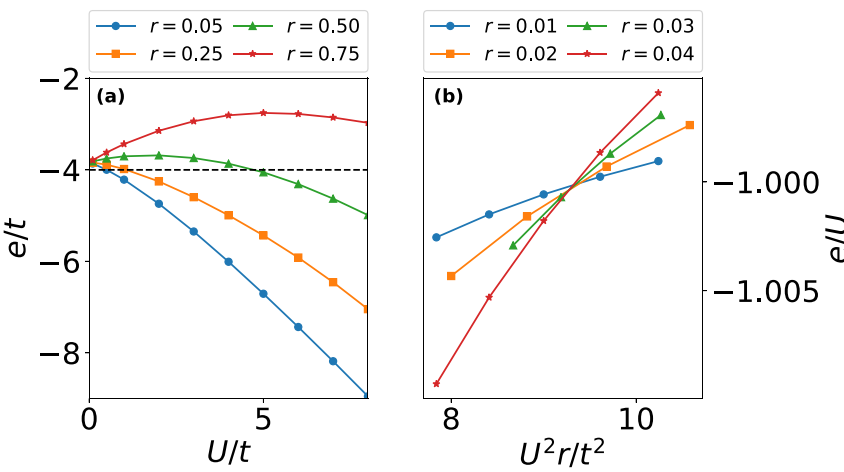

FIG. 1. Energy per particle $e=2 E / N$ in the system with $N=$ 128 particles as a function of the intraspecies interaction strength for different values of $r$ from the weakly interacting situation (a) to the strongly interacting one (b).

we introduce the dimensionless ratio $r=1+U_{A B} / U$, and concentrate on the case of an attractive interspecies interaction $U_{A B}<0$ but always fulfilling $\left|U_{A B}\right|<U(r>0)$. This choice is motivated by previous studies in the continuum geometry, where mean-field interactions were shown to compensate each other exactly for $r=0$ [23]. Furthermore, we will consider open boundary conditions.

From Mott insulator to pair superfluid droplets. The Hamiltonian (1) at strong coupling $U \gg t$ supports different quantum phases, including the Mott insulator (MI) and the pair superfluid (PSF) [26,27]. The PSF is a state characterized by the formation of pairs of atoms (molecules) which exhibit long-range phase coherence [26-29]. On the other hand, coherence is exponentially lost in the MI state. As predicted in Ref. [28], the transition from the MI state into the PSF takes place at a fixed value of $U^{2} r / t^{2}$ independent of the $r$ [see Fig. 1(b)]. We numerically extract the position of the critical point for the MI-PSF transition and obtain $\left(U^{2} r / t^{2}\right)_{c} \simeq 9.25$, shown with the dashed line in Fig. 4. Superfluid states are commonly characterized by having long-range phase coherence. Therefore, we test if the one- (two-) body correlation functions possess off-diagonal quasi-long-range order, seen as a slow power-law decay, and interpret its presence as the superfluidity of atoms (pairs). In addition, we have verified for several selected points that the PSF phase possesses a finite gap $\Delta=E(N+1, N)-2 E(N, N)+E(N-1, N)>0$ which instead is absent in other phases [30]. Thus, the PSF is characterized by (i) the absence of phase coherence in the one-body correlator, (ii) the appearance of phase coherence in the two-body correlator, and (iii) a finite gap associated with spin excitations. Therefore, the correlations between bosons of the same species decay exponentially with the distance $\left\langle\hat{a}_{i} \hat{a}_{j}^{\dagger}\right\rangle=\left\langle\hat{b}_{i} \hat{b}_{j}^{\dagger}\right\rangle \propto \exp \{|i-j| / \xi\}$ [see the $U / t=20$ line in Fig. 2(a)]. Simultaneously, in the PSF phase the correlation function of pairs should behave as $\left\langle\hat{a}_{i} \hat{b}_{i} \hat{a}_{j}^{\dagger} \hat{b}_{j}^{\dagger}\right\rangle \propto 1 /|i-j|^{\alpha}$ [27] [see the $U / t=20$ line in Fig. 2(b)]. Finally, for the MI state all correlation functions decay exponentially (see the $U / t=35$ line in Fig. 2). The change of the form of the decay from exponential in the MI phase to algebraic in the PSF one allows one to identify the phase transition (see Fig. 4).

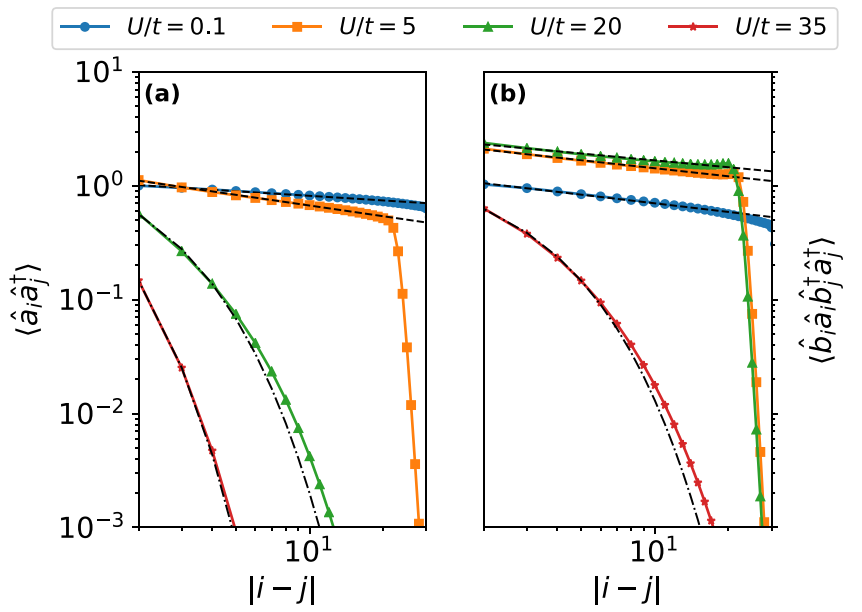

FIG. 2. Correlation functions as a function of the distance $|i-j|$. The index $i=32$ is fixed at the center of the lattice of size $L=64$. Dots correspond to numerical data and dashed (dotted-dashed) lines correspond to algebraic (exponential) fits. For the droplet configurations $(U / t=5,20)$, an abrupt exponential decay is visible in the correlation functions at the edges of the drop. In all cases, $r=0.01$.

An intrinsic property of a dropletlike liquid is being selfbound and localized at zero pressure while a gas occupies the whole available volume. Thus, the density profile contains additional information on the phases (see the inset of Fig. 3 for some examples). For certain parameter values, self-bound objects are observed which do not occupy the whole available space. To characterize these objects, we fit the total density with a symmetrized Fermi function [31] which produces a flattop profile of size $R$ and an exponential decay with a typical length scale $a$ at the edges,

$$
n_{i}=\frac{n_{M} \sinh [R /(2 a)]}{\cosh [R /(2 a)]+\cosh (i / a)},
$$

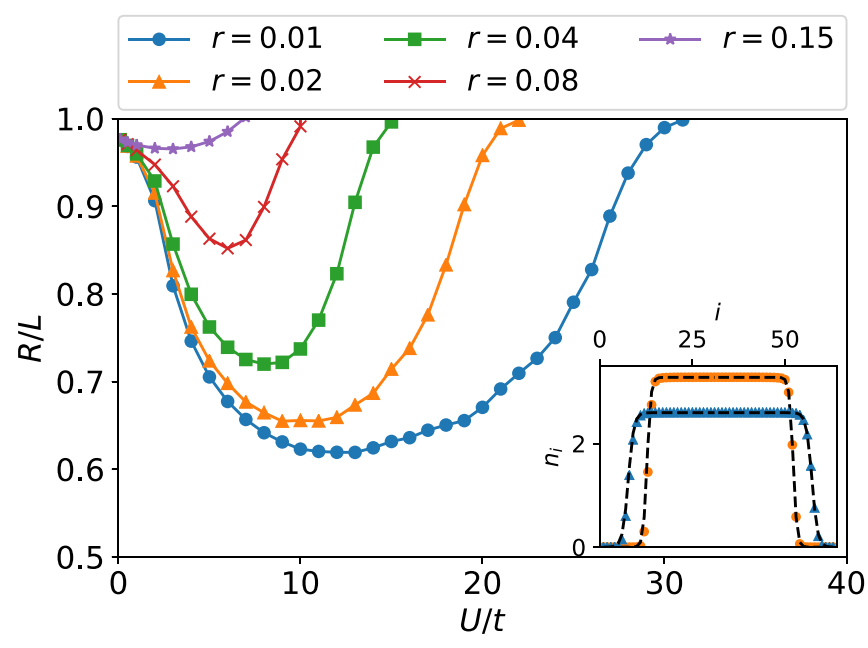

FIG. 3. Typical size of the system as a function of the intraspecies interaction for different ratios $r$, for $N_{A}=N_{B}=L=64$. Inset: Total density profiles of a droplet configuration (dots) for two different interaction strengths $U / t=15,25$ for $r=0.01$. The dashed lines are fits (2) to the density profiles. 


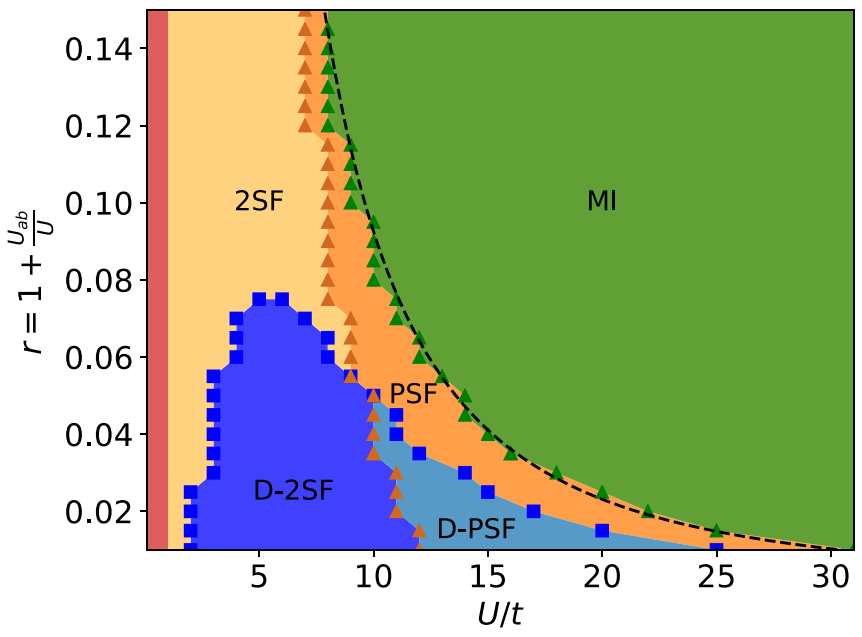

FIG. 4. Phase diagram of the two-component Bose-Hubbard model Eq. (1) close to the droplet regime for $N_{A}=N_{B}=L=64$. The MI phase is characterized by the exponential loss of phase coherence. Green triangles represent the MI-PSF phase transition characterized by the appearance of quasi-long-range coherence in the two-body correlators. The dashed line represents the MI-PSF transition line, $r=9.25 t^{2} / U^{2}$ (see the main text). The PSF-2SF transition (orange triangles) is characterized by the appearance of quasi-long-range coherence in the one- and two-body correlators. Blue squares represent the regime where we detect the appearance of droplets with a size smaller than the lattice length. Droplets are identified by the exponential decay in the density at the edges.

with $n_{M}, R$, and $a$ being free parameters. The size of these droplets has a nontrivial dependence on the interaction $U$ for fixed $r$ as shown in Fig. 3. For any value of $r$, there is a certain value of $U / t$ above which the system is in a MI state, which extends to the full lattice. Decreasing $U / t$, the size of the droplet is found to decrease up to a critical value of $U / t$. Beyond this point, for weaker interactions the droplet size starts to increase until we reach again a size comparable to the system size in the noninteracting limit. In the following, we obtain the phase diagram and clearly identify the important differences between the weakly and strongly interacting regimes.

The phase diagram. The system undergoes dramatic changes when it is brought from the strongly interacting to the weakly interacting regime. Indeed, if we decrease the interaction below the critical value discussed above, we observe that the system goes from a PSF to a two-atomic-superfluid (2SF) state. In the latter, the pair correlator $\left\langle\hat{a}_{i} \hat{b}_{i} \hat{a}_{j}^{\dagger} \hat{b}_{j}^{\dagger}\right\rangle$ and the two correlators $\left\langle\hat{a}_{i} \hat{a}_{j}^{\dagger}\right\rangle$ and $\left\langle\hat{b}_{i} \hat{b}_{j}^{\dagger}\right\rangle$ exhibit a slow power-law decay, meaning that each species separately features quasilong-range phase coherence (see, for instance, the $U / t=5$ line in Fig. 2). Thus, we can now draw two critical lines denoting the quantum phase transition from MI to PSF and from PSF to 2SF (see the phase diagram in Fig. 4). At the same time, a PSF-2SF transition can occur when both phases are in their gaseous form or in their droplet configuration (termed D-PSF and D-2SF). The region of parameters $U / t-r$ in the phase diagram where droplet configurations (smaller than the system size) are stable is also represented in Fig. 4. Due to the open boundary conditions the gaseous phases do not
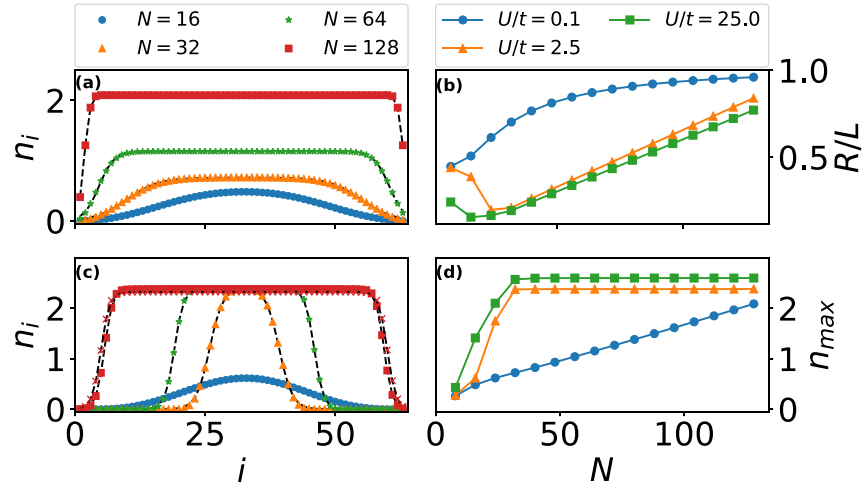

FIG. 5. (a) and (c) Total density profiles $n_{i}$ for weak interactions with $r=0.01$ and several values of $N$. Two cases are considered, a gaseous $2 \mathrm{SF}$ state, $U / t=0.1$, and a D-2SF, $U / t=2.5$ [see the dots in (a) and (c), respectively]. An asymmetric configuration with $U_{A A}=2 U_{B B}=5$ is shown with red crosses in (c). Black dashed lines correspond to fits using Eq. (2). (b) and (d) Droplet properties for a gaseous $2 \mathrm{SF} U / t=0.1$, a D-2SF $U / t=2.5$, and a D-PSF $U / t=25$. The typical spatial size and the maximum density of the system is shown in (b) and (d), respectively.

entirely occupy the system size, because usually the extension is larger than $R>0.95 L$. Droplets are identified when an exponential decay of the density is found at its edges and the size $R$ is smaller than the one observed in the gaseous phase. Importantly, one expects that the Andreev-Bashkin drag [32] is maximal in the 2SF phase in the vicinity of the transition to PSF [33,34]. In this case, a superflow imposed on one component induces a supercurrent in the second component which is dragged without any energy dissipation.

We observe that in the 2SF phase the energy of the system decreases with increasing value of the interaction $U / t$ up to some critical value of $r$ [see Fig. 1(a)]. Therefore, there is a regime where the energy of the system is larger than the zeropoint one, $E_{0}=-2 N t$, where droplet configurations are not stable. This corresponds to the $U / t \lesssim 1$ region in the phase diagram (see Fig. 4).

Quantum droplet properties. Above we have characterized the properties of the ground state for the important case of an integer filling, $N_{A}=N_{B}=L$. Here, we study how the properties change with the filling fraction in a symmetric mixture, $N_{A}=N_{B} \equiv N / 2$. We consider three characteristic examples taken from different phases: a gaseous 2SF, a 2SF droplet, and a PSF droplet. For the gaseous configuration, atoms tend to occupy the whole available space for any number of particles $N$ [see Fig. 5(a)]. When the number of particles $N$ is augmented, the maximum density $n_{\max }$ and the typical extension $R / L$ always increase [see the $U / t=0.1$ line in Fig. 5(d)]. The effect of the open boundary conditions is reflected in the gaseous phases where a decay of the density is observed at the boundaries of the system. This decay does not correspond to an exponential one and it is not captured by Eq. (2). On the other hand, for the droplets a different tendency is observed allowing one to differentiate two additional regimes: small and saturated droplets. In the first regime (small number of particles) the droplets are weakly bound and have a large spatial size. An increase in the number of particles results 


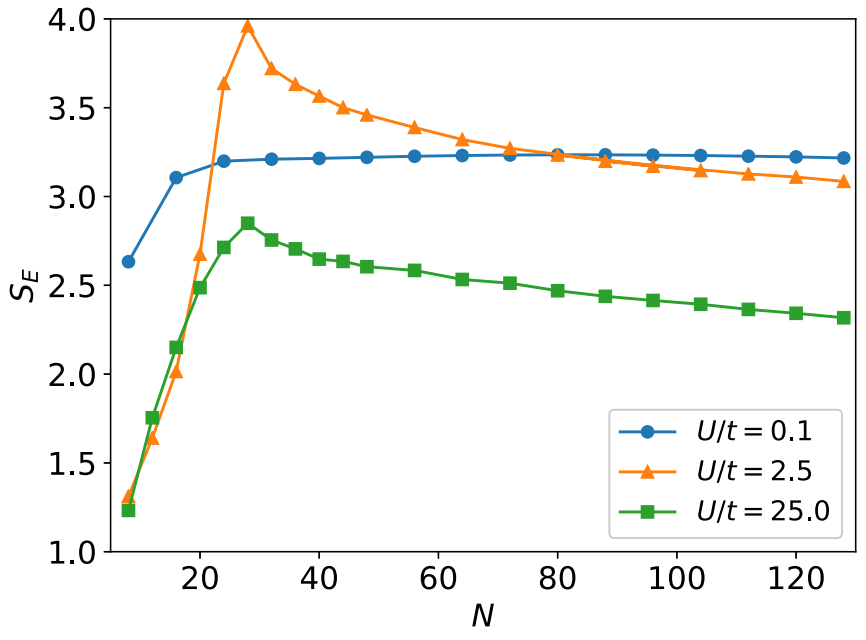

FIG. 6. Entanglement entropy as a function of the total number of particles. We fixed the ratio $r=0.01$ and we choose three interaction strengths $U / t=0.1,2.5,25.0$ corresponding to a gaseous twosuperfluid, a droplet two-superfluid, and a droplet pair superfluid, respectively.

in a stronger binding, decreasing the size, and increasing the maximal density [see the data for $U / t=2.5$ and 25 in Figs. 5(b) and 5(d)]. Then, once the density in the center of the droplet reaches the equilibrium density of the homogeneous liquid, the density saturates and a flattop plateau is formed. In this second regime (large number of particles) the size of the droplets increases for an increasing number of particles, while the maximum density remains constant and equal to the equilibrium density. In both regimes the droplets feature an exponential decay of their density profiles at their boundaries. A notable difference between 2SF and PSF droplets is seen in the small $N$ behavior: The size of PSF droplets drops abruptly as $N$ is increased, while for $2 \mathrm{SF}$ the behavior is smoother, as can be seen comparing the $U / t=2.5$ and $U / t=25$ lines in Fig. 5(b).

Finally, we explore the quantum entanglement present in this system. We consider equal bipartitions of the state and explore the left $(\mathrm{L})$-right $(\mathrm{R})$ entanglement entropy, defined as $S_{E}=-\operatorname{Tr}\left\{\rho_{\mathrm{L}} \log \rho_{\mathrm{L}}\right\}$, where $\rho_{\mathrm{L}}=\operatorname{Tr}_{\mathrm{R}} \rho$ is the reduced density matrix. In Fig. 6 we show the entanglement entropy corresponding to these bipartitions as a function of the total number of particles $N$ and for three characteristic values of the interaction. For the gaseous state we observe that the entanglement entropy saturates as the number of particles is increased. Instead, for the droplet configurations the regimes of saturated and nonsaturated droplets can be distinguished.
For a small number of particles the entanglement entropy rapidly increases with the number of particles. Then it reaches a maximum at the same point where the droplets show a minimum size, shown in Fig. 5. For a larger number of particles the entanglement entropy decreases. Therefore, we can conclude that the droplets with a minimum size for a given number of particles are the ones showing larger quantum effects. It is interesting to note that this is exactly the regime of the strongest correlations where the maximum frequency of the breathing mode in the absence of the lattice is reached [24].

Notes on a possible experimental implementation. One of the main complications of the experimental observation of quantum droplets is their very short lifetimes due to three-body losses $[16,17]$. This effect can be suppressed by reducing the density of the quantum droplet [17]. In our system we have found quantum droplets with densities below three for which three-body losses are greatly suppressed. Another important aspect to take into account for a possible experimental implementation is the asymmetry between the two bosonic species $[14,17]$. To this aim, we have performed numerical simulations introducing an asymmetry between the intraspecies interaction strengths $U_{A A} / t=2 U_{B B} / t$. In this case, the droplet remains stable and the density remains below three (see Fig. 5). All this together makes the system under study very suitable for the possible experimental observation of long-lived quantum droplets.

In conclusion, we have demonstrated the existence of quantum droplets in two-component one-dimensional BoseHubbard chains. We have obtained the phase diagram in the relevant parameter region where multiple phases are realized as a function of the intra- and interspecies interactions. Exploring the long-range decay of one- and two-body correlation functions we have been able to identify quantum droplets with atomic or pair superfluid phase coherence. We have determined a parameter region where three-body recombination effects are negligible, thus opening a way to produce long-lived bosonic droplets. Finally, we have found that the bipartite entanglement entropy present in the drops reaches a maximum when the central density saturates to the equilibrium one and then it decreases for increasing number of particles.

Acknowledgments. The authors thank Leticia Tarruell for useful comments and discussions and Joan Martorell for a careful reading of the manuscript. This work is partially funded by MINECO (Spain) Grants No. FIS201787534-P and No. FIS2017-84114-C2-1-P and from the European Union Regional Development Fund within the ERDF Operational Program of Catalunya (project QUASICAT/QuantumCat).
[1] I. Bloch, J. Dalibard, and W. Zwerger, Rev. Mod. Phys. 80, 885 (2008).

[2] M. Lewenstein, A. Sanpera, and V. Ahufinger, Ultracold Atoms in Optical Lattices: Simulating Quantum Many-Body Systems (Oxford University Press, Oxford, UK, 2012).
[3] S. Sachdev, Quantum Phase Transitions (Cambridge University Press, Cambridge, UK, 2011).

[4] M. Greiner, O. Mandel, T. Esslinger, T. W. Hänsch, and I. Bloch, Nature (London) 415, 39 (2002).

[5] T. Stöferle, H. Moritz, C. Schori, M. Köhl, and T. Esslinger, Phys. Rev. Lett. 92, 130403 (2004). 
[6] E. Haller, R. Hart, M. J. Mark, J. G. Danzl, L. Reichsöllner, M. Gustavsson, M. Dalmonte, G. Pupillo, and H.-C. Nägerl, Nature (London) 466, 597 (2010).

[7] D. S. Petrov, Phys. Rev. Lett. 115, 155302 (2015).

[8] D. Baillie, R. M. Wilson, R. N. Bisset, and P. B. Blakie, Phys. Rev. A 94, 021602(R) (2016).

[9] F. Wächtler and L. Santos, Phys. Rev. A 94, 043618 (2016).

[10] Y. V. Kartashov, G. E. Astrakharchik, B. A. Malomed, and L. Torner, Nat. Rev. Phys. 1, 185 (2019).

[11] I. Ferrier-Barbut, H. Kadau, M. Schmitt, M. Wenzel, and T. Pfau, Phys. Rev. Lett. 116, 215301 (2016).

[12] M. Schmitt, M. Wenzel, F. Böttcher, I. Ferrier-Barbut, and T. Pfau, Nature (London) 539, 259 (2016).

[13] L. Chomaz, S. Baier, D. Petter, M. J. Mark, F. Wächtler, L. Santos, and F. Ferlaino, Phys. Rev. X 6, 041039 (2016).

[14] C. R. Cabrera, L. Tanzi, J. Sanz, B. Naylor, P. Thomas, P. Cheiney, and L. Tarruell, Science 359, 301 (2018).

[15] P. Cheiney, C. R. Cabrera, J. Sanz, B. Naylor, L. Tanzi, and L. Tarruell, Phys. Rev. Lett. 120, 135301 (2018).

[16] G. Semeghini, G. Ferioli, L. Masi, C. Mazzinghi, L. Wolswijk, F. Minardi, M. Modugno, G. Modugno, M. Inguscio, and M. Fattori, Phys. Rev. Lett. 120, 235301 (2018).

[17] C. D'Errico, A. Burchianti, M. Prevedelli, L. Salasnich, F. Ancilotto, M. Modugno, F. Minardi, and C. Fort, Phys. Rev. Research 1, 033155 (2019).

[18] M. Barranco, R. Guardiola, S. Hernández, R. Mayol, J. Navarro, and M. Pi, J. Low Temp. Phys. 142, 1 (2006).

[19] T. Kinoshita, T. Wenger, and D. S. Weiss, Phys. Rev. Lett. 95, 190406 (2005).
[20] D. M. Gangardt and G. V. Shlyapnikov, Phys. Rev. Lett. 90, 010401 (2003).

[21] G. E. Astrakharchik and S. Giorgini, Phys. Rev. A 68, 031602(R) (2003).

[22] B. Laburthe Tolra, K. M. O’Hara, J. H. Huckans, W. D. Phillips, S. L. Rolston, and J. V. Porto, Phys. Rev. Lett. 92, 190401 (2004).

[23] D. S. Petrov and G. E. Astrakharchik, Phys. Rev. Lett. 117, 100401 (2016).

[24] G. E. Astrakharchik and B. A. Malomed, Phys. Rev. A 98, 013631 (2018).

[25] J. Hauschild and F. Pollmann, SciPost Phys. Lect. Notes 5 (2018), doi:10.21468/SciPostPhysLectNotes.5.

[26] A. Kuklov, N. Prokof'ev, and B. Svistunov, Phys. Rev. Lett. 92, 050402 (2004).

[27] A. Hu, L. Mathey, I. Danshita, E. Tiesinga, C. J. Williams, and C. W. Clark, Phys. Rev. A 80, 023619 (2009).

[28] A. Kuklov, N. Prokof'ev, and B. Svistunov, Phys. Rev. Lett. 92, 030403 (2004).

[29] L. Radzihovsky, J. Park, and P. B. Weichman, Phys. Rev. Lett. 92, 160402 (2004).

[30] G. E. Astrakharchik, K. V. Krutitsky, M. Lewenstein, and F. Mazzanti, Phys. Rev. A 93, 021605(R) (2016).

[31] D. W. L. Sprung and J. Martorell, J. Phys. A 30, 6525 (1997).

[32] A. F. Andreev and E. P. Bashkin, Sov. Phys. JETP 42, 164 (1976).

[33] J. Nespolo, G. E. Astrakharchik, and A. Recati, New J. Phys. 19, 125005 (2017).

[34] L. Parisi, G. E. Astrakharchik, and S. Giorgini, Phys. Rev. Lett. 121, 025302 (2018). 